\title{
Article
}

\section{Quantifying varus thrust in knee osteoarthritis using wearable inertial sensors: a proof of concept}

Costello, Kerry E, Eigenbrot, Samantha, Geronimo, Alex, Guermazi, Ali, Felson, David T, Richards, James and Kumar, Deepak

Available at http://clok.uclan.ac.uk/35882/

Costello, Kerry E, Eigenbrot, Samantha, Geronimo, Alex, Guermazi, Ali, Felson, David T, Richards, James ORCID: 0000-0002-4004-3115 and Kumar, Deepak (2020) Quantifying varus thrust in knee osteoarthritis using wearable inertial sensors: a proof of concept. Clinical Biomechanics, 80 (105232). ISSN 02680033

It is advisable to refer to the publisher's version if you intend to cite from the work. http://dx.doi.org/10.1016/j.clinbiomech.2020.105232

For more information about UCLan's research in this area go to http://www.uclan.ac.uk/researchgroups/ and search for < name of research Group>.

For information about Research generally at UCLan please go to http://www.uclan.ac.uk/research/

All outputs in CLoK are protected by Intellectual Property Rights law, including Copyright law. Copyright, IPR and Moral Rights for the works on this site are retained by the individual authors and/or other copyright owners. Terms and conditions for use of this material are defined in the policies page.

\section{CLoK}

Central Lancashire online Knowledge www.clok.uclan.ac.uk

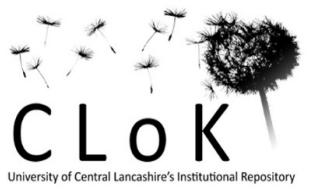


1 Title: Quantifying varus thrust in knee osteoarthritis using wearable inertial sensors: a proof of concept

3 Authors: *Kerry E. Costello, PhD ${ }^{\mathrm{a}, \mathrm{b}}$, *Samantha Eigenbrot, $\mathrm{MS}^{\mathrm{a}}$, Alex Geronimo, MS ${ }^{\mathrm{a}}$, Ali Guermazi, MD,

$4 \quad \mathrm{PhD}^{\mathrm{b}, \mathrm{c}}$, David T. Felson, MD, $\mathrm{MPH}^{\mathrm{a}, \mathrm{b}}$, Jim Richards, $\mathrm{PhD}^{\mathrm{d}}$, Deepak Kumar, $\mathrm{PT}, \mathrm{PhD}^{\mathrm{a}, \mathrm{b}}$

5

6 Affiliations:

$7 \quad{ }^{a}$ Department of Physical Therapy \& Athletic Training, Boston University College of Health \&

8 Rehabilitation Sciences: Sargent College, Boston, MA, USA

$9{ }^{b}$ Division of Rheumatology, Department of Medicine, Boston University School of Medicine, Boston, MA, 10 USA

11 'Department of Radiology, VA Boston Healthcare System, Boston University School of Medicine, Boston, 12 MA, USA

13 d Allied Health Research Unit, School of Sport and Health Sciences, University of Central Lancashire, 14 Preston, UK

15

16 Corresponding author: Deepak Kumar, 635 Commonwealth Ave, Boston MA, 02215, USA,

17 kumard@bu.edu

18

19 Declarations of interest: Ali Guermazi is Shareholder of BICL, LLC and Consultant to AstraZeneca,

20 MerckSerono, TissueGene, Pfizer, Roche and Galapagos. Other authors declare no conflict of interest.

21

22 Word count (abstract): 249; Word count (main text): 3852 


\section{Abstract}

Background: Varus thrust during walking, visualized as excessive frontal plane knee motion during

25 weight acceptance, is a modifiable risk factor for progression of knee osteoarthritis. However, visual

26 assessment does not capture thrust severity and quantification with optical motion capture is often not

27 feasible. Inertial sensors may provide a convenient alternative to optical motion capture. This proof-ofconcept study sought to compare wearable inertial sensors to optical motion capture for the

29 quantification of varus thrust.

Methods: Twenty-six participants with medial knee osteoarthritis underwent gait analysis at self-

31 selected and fast speeds. Linear regression with generalized estimating equations assessed associations

32 between peak knee adduction velocity or knee adduction excursion from optical motion capture and

33 peak thigh or shank adduction velocity from two inertial sensors on the lower limb. Relationships

34 between inertial measures and peak external knee adduction moment were assessed as a secondary

35 aim.

Findings: Both thigh and shank inertial sensor measures were associated with the optical motion capture

37 measures for both speeds $(P<0.001$ to $P=0.020)$, with the thigh measures having less variability than

38 the shank. After accounting for age, sex, body mass index, radiographic severity, and limb alignment,

39 thigh adduction velocity was also associated with knee adduction moment at both speeds (both $P<$

$40 \quad 0.001)$

41 Interpretation: An inertial sensor placed on the mid-thigh can quantify varus thrust in people with

42 medial knee osteoarthritis without the need for optical motion capture. This single sensor may be

43 useful for risk screening or evaluating the effects of interventions in large samples.

44 Keywords: angular velocity, gyroscope, motion capture, adduction 


\section{Introduction}

46 Knee osteoarthritis (OA) is a leading cause of disability among older adults ${ }^{1}$ and most commonly affects

47 the medial tibiofemoral compartment of the knee joint ${ }^{2}$. While age, sex, genetics, and other nonmodifiable factors have been implicated in OA pathogenesis, gait patterns leading to increased or

49 abnormal biomechanical joint loading also play a role and are frequently targeted in interventions ${ }^{3}$. A common gait abnormality in people with medial knee OA is varus thrust, an excessive 'bowing-out' knee

51 motion in the frontal-plane during ambulation as the limb accepts weight with a return towards a more

52 neutral alignment in late stance and swing ${ }^{4,5}$. Varus thrust has been reported to be present in $12 \%$ to

$5346 \%$ of individuals with medial knee OA and has been associated with radiographic disease severity ${ }^{6}$ and

54 progression ${ }^{5}$. Cross-sectionally, those with varus thrust have a five-times greater odds for higher pain

55 during walking and standing than those without it ${ }^{7}$. Individuals with knee OA who exhibit varus thrust

56 also exhibit greater peak external knee adduction moments (EKAM) during gait ${ }^{5}$, an indication of medial

57 tibiofemoral load ${ }^{8}$ which has been reported to be a risk factor for future OA progression ${ }^{9}$. Thus,

58 interventions to reduce varus thrust may lead to reduced pain and slow structural worsening in

59 individuals with medial compartment knee OA.

To aid in the development of effective interventions, it is important to accurately and reliably

61 identify the presence of varus thrust. Typically, varus thrust is assessed through a subjective, visual

62 evaluation of walking ${ }^{5-7,10-14}$. While these assessments are used clinically, they only provide a

63 dichotomous categorization (present/absent) without any indication of severity. To overcome this

64 limitation, optical motion capture has been used to objectively quantify biomechanical parameters as

65 surrogate measures of varus thrust ${ }^{5,6,15-21}$, including knee adduction velocity ${ }^{6}$ and knee adduction

66 angular excursion ${ }^{21}$. However, while optical motion capture provides detailed information on joint

67 kinematics and kinetics, these systems require expensive equipment, time-consuming data collections

68 run by skilled technicians, and a large calibrated measurement volume, making their clinical use 
infeasible. Additionally, analyses conducted in a laboratory environment do not always reflect typical walking in real-world settings ${ }^{22}$. In contrast, small, low-cost wearable inertial sensors have become increasingly popular for collecting biomechanical data in free-living conditions and may provide a

72 convenient alternative to optical motion capture systems for quantifying varus thrust ${ }^{23}$.

The primary aim of this study was to compare data from a single wearable inertial sensor to surrogate measures of varus thrust captured using optical motion capture technology during selfselected and fast speed walking in individuals with medial compartment knee osteoarthritis. We hypothesized that measures of frontal plane segment velocity from single inertial sensors placed on the thigh or shank would be significantly associated with measures from optical motion capture based on previously reported agreement between inertial sensor and optical motion capture kinematic and kinetic measures ${ }^{24,25}$. For a secondary aim, we hypothesized that the inertial sensor measures would be associated with EKAM after adjusting for confounders.

\section{Methods}

\subsection{Participants}

Participants were recruited using advertisements online and in local newspapers from October 2017 to May 2019. Inclusion criteria were age between $45-80$ years, body mass index $(\mathrm{BMI}) \leq 40 \mathrm{~kg} / \mathrm{m}^{2}$, and at least one knee meeting the American College of Rheumatology clinical or radiographic criteria for knee $\mathrm{OA}^{26}$ with primarily medial tibiofemoral compartment involvement (medial joint space narrowing identified from weight bearing knee radiographs). Exclusion criteria were regular use of a walking aid, inflammatory arthritis, lower limb total joint replacement, neurological conditions, muscular disease, or other conditions/treatments affecting gait. This study was approved by the Boston University Institutional Review Board, Boston, USA, and all individuals provided written informed consent prior to radiographic screening and data collection. 


\subsection{Radiographs and Assessment of Symptoms}

95 All participants underwent three radiographs: (1) bilateral weight-bearing posterior-anterior flexed knee radiograph using a Synaflexer positioning frame (BioClinica, Princeton, NJ, USA) for assessment of

97 Kellgren-Lawrence grade (KLG) and assessment of medial and lateral tibiofemoral compartment involvement based on the Osteoarthritis Research Society International (OARSI) atlas, (2) bilateral knee sunrise view for assessment of patellofemoral involvement, and (3) bilateral standing long limb view for measurement of static mechanical axis alignment. An experienced clinician assessed OA severity (KLG) and determined the most involved compartment (medial or lateral) for each knee. Inter-reader reliability for KLG of 0.79 has been reported previously ${ }^{27}$. Static alignment was calculated using OsiriX open-source software (www.osirixviewer.com) ${ }^{28}$ as the angle formed by the intersection of the line

104 between the center of the femoral head and midpoint of the femoral epicondyles, and the line between 105 the midpoint of the femoral epicondyles and midpoint of the malleoli. All participants also completed 106 the Knee Injury and Osteoarthritis Outcome Score $(K O O S)^{29}$. All knees with KLG $<2$ were excluded from 107 the analyses.

\subsection{Optical motion capture}

110 Ground reaction force (GRF) and kinematic data were collected from both legs for all participants while 111 walking along a 15-meter walkway at self-selected and fast speeds. A passive optical motion capture 112 system with 12 infrared cameras and one video camera (Qualisys Medical, Gothenburg, Sweden) was 113 used to capture kinematic data at $250 \mathrm{~Hz}$. Twenty-six spherical retroreflective markers were attached to 114 bony landmarks of the trunk (manubrium, C7 spinous process, T8 spinous process, and the right and left 115 acromion), pelvis (right and left anterior superior iliac spines, superior aspects of iliac crests, right and 116 left posterior superior iliac spines, and sacrum), and lower extremities (greater trochanters, lateral and 
117 medial femoral epicondyles, lateral and medial malleoli, first and fifth metatarsal heads) which were

118 used to identify joint centers during a static standing trial (Figure 1A). Rigid clusters of four markers

119 were placed on the shank and thigh segments, and markers were placed on the posterior aspect of the

120 heel, medial and lateral mid-foot, $2^{\text {nd }}$ metatarsal head, and lateral aspect of $5^{\text {th }}$ metatarsal head for

121 segment tracking (Figure 1A). Three force platforms (AMTI, Watertown, MA, USA) were used to collect

122 GRF data at $2000 \mathrm{~Hz}$ synchronously with the motion data. Walking speed was measured using a timing

123 system (Brower Timing Systems, Draper, UT, USA). Self-selected and fast walking target speeds were

124 determined after 3-4 practice trials. For self-selected speed trials, participants were instructed to "walk

125 across the room towards the door with a purposeful pace." For fast walking trials, participants were

126 instructed to "walk as quickly as you comfortably can without running and without going faster than

127 what you feel is safe for you." Any trials that were outside $\pm 5 \%$ of the target walking speeds were

128 excluded. Four to six clean force plate foot strikes were collected for each foot. Each participant wore

129 laboratory-provided shoes (Gel-Cumulus 19, ASICS, Kobe, Japan).

Within the motion capture software, marker trajectories were identified and any gaps in data

131 were filled using polynomial splines for gaps of less than 10 frames and trajectory matching for larger

132 gaps. All data were then imported into Visual3D (C-Motion, Germantown, MD, USA). Marker and GRF

133 data were filtered using low-pass, 4th order Butterworth filters with cut-off frequencies of $6 \mathrm{~Hz}$ and 12

$134 \mathrm{~Hz}$, respectively, as the majority of movement during walking occurs below these frequencies. Joint

135 kinematics were calculated from the marker data using Euler angles ( $x-y-z)$ and right-handed coordinate

136 systems. Initial and final contact for each clean foot strike were identified from the GRF using a

137 threshold of $20 \mathrm{~N}$ to define stance phase.

The primary measure from the optical motion capture system was peak knee adduction velocity

139 and the secondary measure was knee adduction excursion. Chang et al. suggested peak knee adduction

140 velocity as an appropriate biomechanical index to quantify varus thrust as it closely corresponded with 
141 visual assessments and captures both the direction and speed of movement ${ }^{6}$. As the majority of the

142 varus thrust movement occurs in early stance ${ }^{6}$, peak knee adduction velocity during the first half of

143 stance phase was recorded (Figure 2B). It should be noted that because the joint angle (Euler angle) is a

144 vector quantity, it is not possible to compute the joint angular velocity by taking the first derivative of

145 the joint angle ${ }^{30}$. We used $X-Y-Z$ cardan sequence to calculate the knee joint angle (shank relative to

146 thigh); the knee joint angular velocity was calculated as the angular velocity of the shank relative to the

147 thigh ${ }^{30,31}$. The secondary motion capture varus thrust measure, knee adduction excursion, was

148 calculated as the difference between the knee adduction angle at initial contact and the maximum knee 149 adduction angle during the first half of stance $^{6}$ (Figure 2 C). Inverse dynamics were used to calculate the 150 EKAM, normalized to body weight and height (\% bodyweight-height), and the peak EKAM was extracted 151 from the first half of stance (Figure 2D). All variables were averaged across all clean foot strikes for each 152 leg.

155 Small, lightweight inertial sensors (Trigno ${ }^{\mathrm{TM}}$ IM Sensor, Delsys, Inc., Natick, MA, USA) were used 156 concurrently with the optical motion capture system. Each sensor measured $37 \mathrm{~mm} \times 26 \mathrm{~mm} \times 15 \mathrm{~mm}$, 157 weighed $14.7 \mathrm{~g}$, and consisted of a triaxial accelerometer $( \pm 16 \mathrm{~g})$, a triaxial gyroscope $( \pm 2000 \mathrm{degree} / \mathrm{s})$, 158 and a triaxial magnetometer $( \pm 1000 \mu \mathrm{T})$. Initially, three sensor locations were tested for each limb 159 (Figure 1B): 1) lateral mid-thigh (attached to the thigh segment optical motion capture marker cluster), 160 2) lateral mid-shank (attached to the shank segment optical motion capture marker cluster), and 3) 161 lateral distal shank (attached directly to the skin on the lateral aspect of the distal tibia proximal to the 162 lateral malleolus). The distal shank placement was included with the assumption that it would be more 163 convenient for use in a non-laboratory environment. However, interim analyses showed poor 164 association between optical motion capture data and data from the distal sensor, particularly during fast 
speed walking, and this sensor was perceived as uncomfortable by a few participants. For these reasons,

166 use of the distal sensor placement was discontinued for the final 11 participants ( 22 knees) enrolled in

167 this study and the data from this sensor are not presented here. For all sensor placements, while a

168 general orientation of the sensors was specified (i.e. arrow side 'up'), the placement along the length of

169 the leg and anterior-posterior position were not constrained, nor were any calibration procedures

170 performed, in order to better replicate placement of the sensors in a clinical setting and/or by untrained

171 individuals (e.g. participants).

Data were recorded from each sensor at $148 \mathrm{~Hz}$, upsampled to $2000 \mathrm{~Hz}$, and time synchronized

173 with the optical motion capture system. The frontal plane component of the raw gyroscope data was

174 used as a measure of segment adduction velocity. The gyroscope component was chosen as it captures

175 angular velocity as compared to accelerometer components which capture linear acceleration. Peak

176 segment adduction velocity in degrees per second, which was calculated as the peak value between

177 initial contact and midstance, was extracted for each trial for each sensor (Figure 2A) and averaged

178 across trials for each leg. Outcomes used in the analyses included peak thigh adduction velocity from the

179 mid-thigh sensor (mid-thigh adduction velocity) and peak shank adduction velocity from the mid-shank

180 sensor (mid-shank adduction velocity) for each walking speed. It should be noted that the thigh

181 adduction velocity is recorded as positive and shank adduction velocity is recorded as negative (Figure 2,

182 Table 2) given the nature of segmental motion and orientation of the sensor coordinate systems (Figure 183 1B).

\subsection{Statistical Analyses}

186 For the primary aim, univariate regression models with generalized estimating equations (GEE) were

187 used to assess the relationships between the inertial sensor measures and the optical motion capture 188 measures, separately for self-selected and fast speed walking. The GEEs allowed us to account the 
correlation between knees within each person. For the secondary aim, multivariate regression models

with GEE were used to assess the relationships between EKAM and the inertial sensor measures, separately for each speed, while adjusting for a number of confounders (specifically age, sex, BMI, KLG, and static alignment) that may affect both varus thrust ${ }^{13}$ and $\mathrm{EKAM}^{32-34}$. While pain can also affect EKAM $^{32}$, we hypothesized that it acts as a mediator rather than a confounder on the causal pathway (i.e. varus thrust causes pain rather than the other way around), and thus did not include it in the models. All models were constructed including a term for leg and an interaction term between exposure and leg to determine whether the association of the exposure and the outcome differed by leg. If there was no interaction between exposure and leg (i.e. this interaction term was not significant), the model was re-run without the exposure by leg interaction term. All analyses were performed using IBM SPSS

199 (Armonk, NY, USA) in all knees with KLG $\geq 2$. Significance was set at $\alpha=0.05$.

\section{Results}

One hundred and sixty-three individuals underwent telephone screening, 82 passed the initial screening process, 59 underwent a radiographic screening visit, and 26 individuals (16 female) were deemed eligible for this study (Figure 3, Table 1). The number of knees across analyses differed depending on useable data available for each inertial sensor (Figure 3). Average values for each inertial and optical motion capture measure are reported in Table 2.

Both mid-thigh and mid-shank adduction velocity were associated with knee adduction velocity and excursion during self-selected and fast speed walking. An increase of $10.0 \% \mathrm{~s}$ in mid-thigh adduction

209 velocity was associated with an increase in knee adduction velocity of $6.1 \%$ s during self-selected speed

210 walking $(P<0.001$, Figure $4 \mathrm{~A})$ and $5.3^{\circ} / \mathrm{s}$ during fast walking $(P=0.001$, Figure 4B), and an increase in

211 knee adduction excursion of $0.35^{\circ}$ during self-selected speed walking $(P=0.005$, Figure $4 C)$ and $0.33^{\circ}$

212 during fast walking $\left(P=0.020\right.$, Figure 4D). Similarly, an increase of $10.0^{\circ} / \mathrm{s}$ in mid-shank adduction 
213 velocity was associated with an increase in knee adduction velocity of $3.4 \%$ s during self-selected speed

214 walking $(P<0.001$, Figure $4 \mathrm{~A})$ and $2.2^{\circ}$ s during fast walking $(P=0.005$, Figure $4 \mathrm{~B})$, and an increase in

215 knee adduction excursion of $0.20^{\circ}$ during both self-selected $(P=0.004$, Figure $4 C)$ and fast $(P<0.001$,

216 Figure 4D) speed walking.

After accounting for age, sex, BMI, KLG, and static alignment, an increase of $10.0 \% \mathrm{~s}$ in mid-thigh

218 adduction velocity was associated with an increase in EKAM of $0.16 \%$ bodyweight-height $(P<0.001)$

219 during self-selected speed walking and an increase of $0.17 \%$ bodyweight-height $(P<0.001)$ during fast

220 speed walking. For the models investigating the relationship between mid-shank adduction velocity and

221 EKAM during self-selected and fast speed walking, the interaction term between leg and mid-shank

222 adduction velocity was significant, thus separate models were run for left and right legs. For left legs $(n=$

223 18), an increase in $10.0 \%$ in mid-shank adduction velocity was associated with an increase in EKAM of

$224 \quad 0.10 \%$ bodyweight-height $(P=0.010)$ during self-selected speed walking and an increase in $0.17 \%$

225 bodyweight-height $(P<0.001)$ during fast speed walking, after accounting for confounders. For right legs

$226(n=23)$, mid-shank adduction velocity was not associated with EKAM at either self-selected speed $(P=$

$2270.88)$ or fast speed $(P=0.21)$.

228

229 4. Discussion

230 This proof-of-concept study showed that the measures from single inertial sensors were associated with

231 surrogate measures of varus thrust obtained using optical motion capture. Furthermore, supporting our

232 secondary hypothesis, mid-thigh adduction velocity was significantly associated with peak EKAM after

233 adjusting for confounders. These results suggest that inertial sensors should be further investigated as a

234 tool to objectively quantify varus thrust in clinical settings where optical motion capture is not feasible

235 and visual assessment is insufficient. The ability to quickly and accurately quantify varus thrust in clinical 
or other real-world settings could lead to better identification and treatment of those at risk of OA

237 progression due to varus thrust.

Both inertial sensor metrics - mid-thigh adduction velocity and mid-shank adduction velocity were associated with both optical motion capture thrust measures at both walking speeds. The mid-

240 shank data, however, had greater variability than the mid-thigh data, e.g. an adduction velocity range of $241165 \%$ for the mid-shank versus $99 \%$ s for the mid-thigh during self-selected speed walking (Figure 4,

242 Table 2), suggesting that the mid-thigh sensor placement is superior. These results are supported by 243 previous research that found data from a single mid-thigh inertial sensor were a better predictor of peak 244 knee extensor moment and power absorption during a single limb task than a mid-shank sensor ${ }^{35}$ and 245 that data from a single thigh accelerometer, but not a single shank accelerometer, were predictive of 246 between-limb differences in knee power absorption during running ${ }^{36}$.

In the current study, both of the mid-segment inertial sensors were placed directly on the rigid optical motion capture marker clusters used for segment tracking. However, it is the anatomical coordinate system defined by markers placed on bony landmarks, rather than these rigid clusters, that 250 define the segment coordinate systems for the motion capture measures. Orientation of the inertial 251 sensors relative to the anatomical coordinate system is a key factor in accuracy of joint angles measured 252 by inertial sensors ${ }^{37}$. A varying degree of curvature on the lateral aspect of the shank due to the shape of 253 the lateral head of the gastrocnemius muscle could have resulted in less consistent placement of the 254 mid-shank inertial sensor among legs, thus affecting the relationship between the inertial sensor 255 coordinate system and the anatomical coordinate system of the shank. In contrast, the lateral thigh has 256 less variation in curvature along its length, which may have ensured more consistent placement of the 257 mid-thigh inertial sensor across legs. Thus, the mid-thigh sensor placement may be preferable to the 258 mid-shank placement for assessing varus thrust in individuals with knee OA, particularly in cases where 259 limited time or experience prevent more standardized placement. 
The regression models for EKAM also provide support for the mid-thigh versus mid-shank placement as the association between segment angular velocity and EKAM was not consistent across

262 legs for the mid-shank sensor, i.e. it was significant for the left but not the right leg. While the current 263 dataset did not provide enough power for a thorough comparison between left and right legs, these 264 groups were similar in terms of KLG and HKA, thus no difference between legs was expected. Despite 265 this discrepancy between legs for the mid-shank sensor, the novel finding that mid-thigh adduction 266 velocity was significantly associated with EKAM, even after adjusting for confounders, is promising as it 267 suggests that an estimation of EKAM may be possible without the need for extensive laboratory 268 equipment or analysis of multiple inertial sensors. This finding also supports previous studies that have 269 shown associations between various quantitative measures of varus thrust and EKAM ${ }^{17,20,21}$. In the current study, only single-sensor inertial measures were examined with the idea that 271 large-scale screening in a clinical setting would require a quick set-up with minimal data processing.

272 However, the lack of an exact 1:1 increase in adduction velocity between these inertial sensor and

273 optical motion captures measures may be attributed to the fact that the inertial sensor segment

274 adduction velocity measures only describe movement of a single segment (thigh or shank), while knee 275 adduction velocity describes the relative movement between the thigh and shank segments. Integration 276 of multiple inertial sensors or sensor components may result in better estimation of knee adduction

277 velocity, however, this typically requires a series of functional calibration exercises, modeling

278 assumptions, and data filtering to address issues such as drift and sensor alignment ${ }^{38}$. The finding in the 279 current study of a significant association between single inertial sensor measures and optical motion 280 capture measures suggests a single sensor may be sufficient as a quick screening tool for severity of 281 varus thrust in knee OA populations where more extensive data collection is not feasible. We observed significant associations of mid-thigh and mid-shank sensors with both knee 283 adduction velocity and knee adduction excursion measures at both walking speeds. As noted by Chang 
et al. ${ }^{6}$, knee adduction velocity captures the speed of the movement and can be used as a reliable measure of varus thrust in people with knee OA. Knee adduction excursion has also been used as a quantitative metric of varus thrust and has been associated with both OA severity (KLG) and EKAM in

287 individuals with medial knee $\mathrm{OA}^{21}$. Associations would be expected between measures of thigh/shank 288 adduction velocity from the inertial sensors and knee adduction velocity from optical motion capture 289 given the similar constructs being measured. However, significant associations of metrics from thigh/shank inertial sensors and knee adduction excursion further support the use of inertial sensors to 291 assess varus thrust. Furthermore, the similarity of results across the two speeds for the analyses in the current study suggests that a single sensor could be used to quantify varus thrust across different walking speeds in individuals with knee OA.

There are a number of limitations of the current study that should be acknowledged. Given that 295 this was a proof of concept study, the sample size was small. Data error resulted in the loss of inertial 296 sensor data for some legs due to signal clipping. While this appeared to be an issue with software 297 presets, rather than the inertial sensors themselves, the loss of this data and small sample size overall 298 did not provide enough power for a definitive comparison across sensor locations or speeds. The lack of 299 standardized placement of the inertial sensors on the leg, e.g. a specified percent distance along a given 300 segment, may have increased variability in the measurement of segment adduction velocities from the 301 inertial sensors, resulting in misalignment of coordinate systems between the inertial and optical motion 302 capture systems and an inability to provide a definitive recommendation on sensor placement. The 303 attachment of sensors in a clinical setting would likely also be done without standardized placement or 304 calibration and thus these results may be a good representation of how clinical inertial sensor data 305 would correspond to optical motion capture data. It should be noted, however, that the sensors in the current study were placed on top of the rigid plate containing optical motion capture markers and it is 307 unclear whether a rigid sensor alone versus the rigid sensor/plate combination would produce similar 
data. In this study, gait events identified by the optical motion capture system were used for calculation

309 of the inertial measures. In a clinical setting where optical motion capture data are not available, gait

310 events calculated from the inertial sensors, as has been done previously ${ }^{39}$, would need to be utilized.

311 Reliability of the thigh inertial sensor measurement will also need to be established before it can be

312 used in clinical settings, particularly given the limited number of studies investigating reliability of

313 inertial sensors in knee OA populations ${ }^{40,41}$. It should also be noted that while the sample in the current

314 study is similar to that of larger studies of individuals with medial knee OA in terms of age, sex, and

$315 \mathrm{BMI}^{13}$, the results may not generalize to other sub-groups of the OA population such as individuals with

$316 \quad \mathrm{BMI}>40$.

\section{5. Conclusions}

319 In this proof-of-concept study, we demonstrated a significant association between increases in thigh 320 angular velocity derived from the gyroscope signal of a single inertial sensor and increases in surrogate

321 varus thrust measures derived from an optical motion capture system. Furthermore, increased thigh

322 angular velocity from this single inertial sensor was associated with increased peak EKAM after adjusting

323 for confounders. These results highlight the potential of inertial sensors for quantifying varus thrust

324 without the need for an optical motion capture system.

\section{Acknowledgments}

327 We would like to acknowledge the staff and students of the Movement \& Applied Imaging Laboratory

328 for their contributions to data collection and processing. We would also like to thank the participants for 329 their contributions to this study and ASICS for donating the shoes used in this study. 
331 award numbers K01AR069720 and T32AR007598. The content is solely the responsibility of the authors

332 and does not necessarily represent the official views of the National Institutes of Health. 


\section{$\underline{\text { References }}$}

335 1. Guccione A, Felson DT, Anderson JJ, et al. The effects of specific medical conditions on the functional limitations of elders in the Framingham study. Am J Public Health. 1994;84(3):351-358.

337 2. McAlindon TE, Snow S, Cooper C, Dieppe PA. Radiographic patterns of osteoarthritis of the knee joint in the community: The importance of the patellofemoral joint. Ann Rheum Dis. 1992;51:844849. doi:10.1136/ard.51.7.844

3. Felson DT, Lawrence RC, Dieppe PA, et al. Osteoarthritis: New insights - Part 1: The disease and its risk factors. Ann Intern Med. 2000. doi:10.7326/0003-4819-133-8-200010170-00016

4. Wink AE, Gross KD, Brown CA, et al. Varus thrust during walking and the risk of incident and worsening medial tibiofemoral MRI lesions: the Multicenter Osteoarthritis Study. Osteoarthr Cartil. 2017;25(6):839-845.

5. Chang A, Hayes K, Dunlop D, et al. Thrust during ambulation and the progression of knee osteoarthritis. Arthritis Rheum. 2004;50(12):3897-3903. doi:10.1002/art.20657

347 6. Chang AH, Chmiel JS, Moisio KC, et al. Varus thrust and knee frontal plane dynamic motion in persons with knee osteoarthritis. Osteoarthr Cartil. 2013;21(11):1668-1673.

349 7. Lo GH, Harvey WF, McAlindon TE. Associations of varus thrust and alignment with pain in knee osteoarthritis. Arthritis Rheum. 2012;64(7):2252-2259. doi:10.1002/art.34422

351 8. Hurwitz DE, Sumner DR, Andriacchi TP, Sugar DA. Dynamic knee loads during gait predict proximal tibial bone distribution. J Biomech. 1998;31(5):423-430. doi:10.1016/S00219290(98)00028-1

354 9. Chang AH, Moisio KC, Chmiel JS, et al. External knee adduction and flexion moments during gait and medial tibiofemoral disease progression in knee osteoarthritis. Osteoarthr Cartil. 
357 10. lijima H, Fukutani N, Aoyama T, et al. Clinical phenotype classifications based on static varus alignment and varus thrust in Japanese patients with medial knee osteoarthritis. Arthritis Rheumatol. 2015;67(9):2354-2362.

11. lijima H, Fukutani N, Yamamoto Y, et al. Association of varus thrust with prevalent patellofemoral osteoarthritis: A cross-sectional study. Gait Posture. 2017;58:394-400. doi:10.1016/j.gaitpost.2017.08.033

12. Fukutani $\mathrm{N}$, lijima $\mathrm{H}$, Fukumoto $\mathrm{T}$, et al. Association of varus thrust with pain and stiffness and activities of daily living in patients with medial knee osteoarthritis. Phys Ther. 2016;96(2):167175. doi:10.2522/ptj.20140441

13. Chang A, Hochberg M, Song J, et al. Frequency of varus and valgus thrust and factors associated Rheum. 2010;62(5):1403-1411. doi:10.1002/art.27377

14. Sharma L, Chang AH, Jackson RD, et al. Varus thrust and incident and progressive knee osteoarthritis. Arthritis Rheumatol. 2017. doi:10.1002/art.40224

371 15. Fukaya T, Mutsuzaki H, Wadano Y. Kinematic analysis of knee varus and rotation movements at the initial stance phase with severe osteoarthritis of the knee. Knee. 2015;22(3):213-216. doi:10.1016/j.knee.2015.02.012

374 16. Kakihana W, Akai M, Nakazawa K, Naito K, Torii S. Inconsistent knee varus moment reduction 375 caused by a lateral wedge in knee osteoarthritis. Am J Phys Med Rehabil. 2007;86(6):446-454. doi:10.1097/PHM.0b013e31805bfff5

377 17. Mahmoudian A, van Dieen JH, Bruijn SM, et al. Varus thrust in women with early medial knee 
osteoarthritis and its relation with the external knee adduction moment. Clin Biomech.

2016;39:109-114. doi:10.1016/j.clinbiomech.2016.10.006

380

381

382

383

384

18. Brown TN, Kaplan JT, Cameron SE, Seymore KD, Ramsay JW. Individuals with varus thrust do not increase knee adduction when running with body borne load. Journal of Biomechanics. 2018.

19. Hunt MA, Schache AG, Hinman RS, Crossley KM. Varus thrust in medial knee osteoarthritis: Quantification and effects of different gait-related interventions using a single case study. Arthritis Care Res. 2011;63(2):293-297. doi:10.1002/acr.20341

20. Sosdian L, Hinman RS, Wrigley T V., et al. Quantifying varus and valgus thrust in individuals with severe knee osteoarthritis. Clin Biomech. 2016;39:44-51. doi:10.1016/j.clinbiomech.2016.09.007

21. Kuroyanagi $Y$, Nagura $T$, Kiriyama $Y$, et al. A quantitative assessment of varus thrust in patients with medial knee osteoarthritis. Knee. 2012;19(2):130-134. doi:10.1016/j.knee.2010.12.007

22. Brodie MAD, Coppens MJM, Lord SR, et al. Wearable pendant device monitoring using new wavelet-based methods shows daily life and laboratory gaits are different. Med Biol Eng Comput. 2016;54(4):663-674. doi:10.1007/s11517-015-1357-9

23. Tao W, Liu T, Zheng R, Feng H. Gait analysis using wearable sensors. Sensors. 2012;12(2):22552283. doi:10.3390/s120202255

24. Konrath JM, Karatsidis A, Schepers HM, Bellusci G, de Zee M, Andersen MS. Estimation of the Knee Adduction Moment and Joint Contact Force during Daily Living Activities Using Inertial Motion Capture. Sensors. 2019;19. doi:10.3390/s19071681

25. Zügner R, Tranberg R, Timperley J, Hodgins D, Mohaddes M, Kärrholm J. Validation of inertial measurement units with optical tracking system in patients operated with Total hip arthroplasty. BMC Musculoskelet Disord. 2019;20(1). doi:10.1186/s12891-019-2416-4 
26. Altman R, Asch E, Bloch D, et al. Development of criteria for the classification and reporting of osteoarthritis: Classification of osteoarthritis of the knee. Arthritis Rheumatol. 1986;29(8):10391049. doi:10.1002/art.1780290816

27. Guermazi A, Hayashi D, Roemer F, et al. Severe radiographic knee osteoarthritis - does Kellgren and Lawrence grade 4 represent end stage disease? - the MOST study. Osteoarthr Cartil. 2015;23(9):1499-1505. doi:10.1016/j.joca.2015.04.018

28. Rosset A, Spadola L, Ratib O. OsiriX: An open-source software for navigating in multidimensional DICOM images. J Digit Imaging. 2004;17(3):205-216. doi:10.1007/s10278-004-1014-6

29. Roos EM, Roos HP, Lohmander LS, Ekdahl C, Beynnon BD. Knee Injury and Osteoarthritis Outcome Score (KOOS)—Development of a self-administered outcome measure. J Orthop Sport Phys Ther. 1998;28(2):88-96. doi:10.2519/jospt.1998.28.2.88

30. Hamill J, Selbie W, Kepple T. Three-dimensional kinematics. In: Robertson DGE, Caldwell GE, Hamill J, Kamen G, Whittlesey SN, eds. Research Methods in Biomechanics. 2nd ed. Champaign, IL, USA; 2013:35-60.

31. C-Motion. Joint Velocity. https://c-motion.com/v3dwiki/index.php/Joint_Velocity. Accessed October 13, 2020.

32. Hurwitz DE, Ryals AB, Case JP, Block JA, Andriacchi TP. The knee adduction moment during gait in subjects with knee osteoarthritis is more closely correlated with static alignment than radiographic disease severity, toe out angle and pain. J Orthop Res. 2002;20(1):101-107. doi:10.1016/S0736-0266(01)00081-X

33. Blazek K, Asay JL, Erhart-Hledik J, Andriacchi T. Adduction moment increases with age in healthy obese individuals. J Orthop Res. 2013;31(9):1414-1422. doi:10.1002/jor.22390 
422 34. Sims EL, Carland JM, Keefe FJ, Kraus VB, Guilak F, Schmitt D. Sex differences in biomechanics associated with knee osteoarthritis. J Women Aging. 2009;21(3):159-170. doi:10.1080/08952840903054856

35. Pratt KA, Sigward SM. Inertial sensor angular velocities reflect dynamic knee loading during single limb loading in individuals following anterior cruciate ligament reconstruction. Sensors (Switzerland). 2018;18(10). doi:10.3390/s18103460

428

36. Havens KL, Cohen SC, Pratt KA, Sigward SM. Accelerations from wearable accelerometers reflect knee loading during running after anterior cruciate ligament reconstruction. Clin Biomech. 2018;58(July):57-61. doi:10.1016/j.clinbiomech.2018.07.007

37. Kianifar R, Joukov V, Lee A, Raina S, Kulić D. Inertial measurement unit-based pose estimation: Analyzing and reducing sensitivity to sensor placement and body measures. J Rehabil Assist Technol Eng. 2019;6:205566831881345. doi:10.1177/2055668318813455

38. Seel T, Raisch J, Schauer T. IMU-based joint angle measurement for gait analysis. Sensors. 2014;14(4):6891-6909. doi:10.3390/s140406891

39. Aminian K, Najafi B, Büla C, Leyvraz P-F, Robert P. Spatio-temporal parameters of gait measured by an ambulatory system using miniature gyroscopes. J Biomech. 2002;35(5):689-699. doi:10.1016/S0021-9290(02)00008-8

40. Kobsar D, Osis ST, Phinyomark A, Boyd JE, Ferber R. Reliability of gait analysis using wearable sensors in patients with knee osteoarthritis. J Biomech. 2016;49(16):3977-3982. doi:10.1016/j.jbiomech.2016.11.047

41. Turcot K, Aissaoui R, Boivin K, Hagemeister N, Pelletier M, Guis JA d. Test-retest reliability and minimal clinical change determination for 3-dimensional tibial and femoral accelerations during treadmill walking in knee osteoarthritis patients. Arch Phys Med Rehabil. 2008;89(4):732-737. 
446

447 
Table 1. Sample characteristics

\begin{tabular}{|c|c|}
\hline $\begin{array}{c}\text { Clinical \& Demographic } \\
\text { Characteristics }\end{array}$ & $n=26$ participants $t$ \\
\hline Sex & $\mathrm{n}=16[62 \%]$ female \\
\hline Age (years) & $64.5(8.4)$ \\
\hline Body mass index $\left(\mathrm{kg} / \mathrm{m}^{2}\right)$ & $28.6(4.7)$ \\
\hline Height $(\mathrm{m})$ & $1.67(0.12)$ \\
\hline \multicolumn{2}{|l|}{ Gait speed $(\mathrm{m} / \mathrm{s})$ : } \\
\hline Self-selected & $1.32(0.22)$ \\
\hline Fast & $1.62(0.32)$ \\
\hline $\begin{array}{l}\text { Knee Injury and Osteoarthritis } \\
\text { Outcome Score - Pain }\left(/ 100^{*}\right)\end{array}$ & $59.2(11.1)$ \\
\hline \multicolumn{2}{|l|}{ Kellgren-Lawrence Grade (KLG): } \\
\hline $\mathrm{KLG}=2$ & $\mathrm{n}=13$ legs \\
\hline$K L G=3$ & $n=26$ legs \\
\hline $\mathrm{KLG}=4$ & $\mathrm{n}=8$ legs \\
\hline Static limb alignment (degrees) ${ }^{* *}$ & $175.9(3.6)$ \\
\hline
\end{tabular}

Data presented as mean (standard deviation) except where

noted

$t_{n}=47$ knees from these participants had $K L G \geq 2$. KOOS, KLG,

\& alignment values are reported for these knees only.

* Lower scores represent greater pain

**Angles < 180 degrees indicate static varus alignment 
451 Table 2: Inertial and optical motion capture outcome average values in knees with radiographic OA (KLG

$452 \geq 2)$

\begin{tabular}{lc}
\hline Inertial motion capture & \\
\hline Mid-thigh adduction velocity (degree/s) ( $\mathrm{n}=39$ legs): & $45.2(25.2)$ \\
$\quad$ Self-selected speed walking & $58.9(28.1)$ \\
$\quad$ Fast speed walking & $-80.4(36.9)$ \\
Mid-shank adduction velocity (degree/s) ( $\mathrm{n}=41$ legs): & $-97.3(43.4)$ \\
$\quad$ Self-selected speed walking & \\
$\quad$ Fast speed walking & \\
\hline Optical motion capture & $63.9(24.8)$ \\
\hline Knee adduction velocity (degree/s) ( $\mathrm{n}=45$ legs): & $75.6(28.6)$ \\
$\quad$ Self-selected speed walking & $3.8(2.0)$ \\
$\quad$ Fast speed walking & $4.0(2.1)$ \\
Knee adduction excursion (degree) ( $\mathrm{n}=45$ legs): & 35 legs): \\
$\quad$ Self-selected speed walking & $3.25(1.22)$ \\
$\quad$ Fast speed walking &
\end{tabular}


A

B

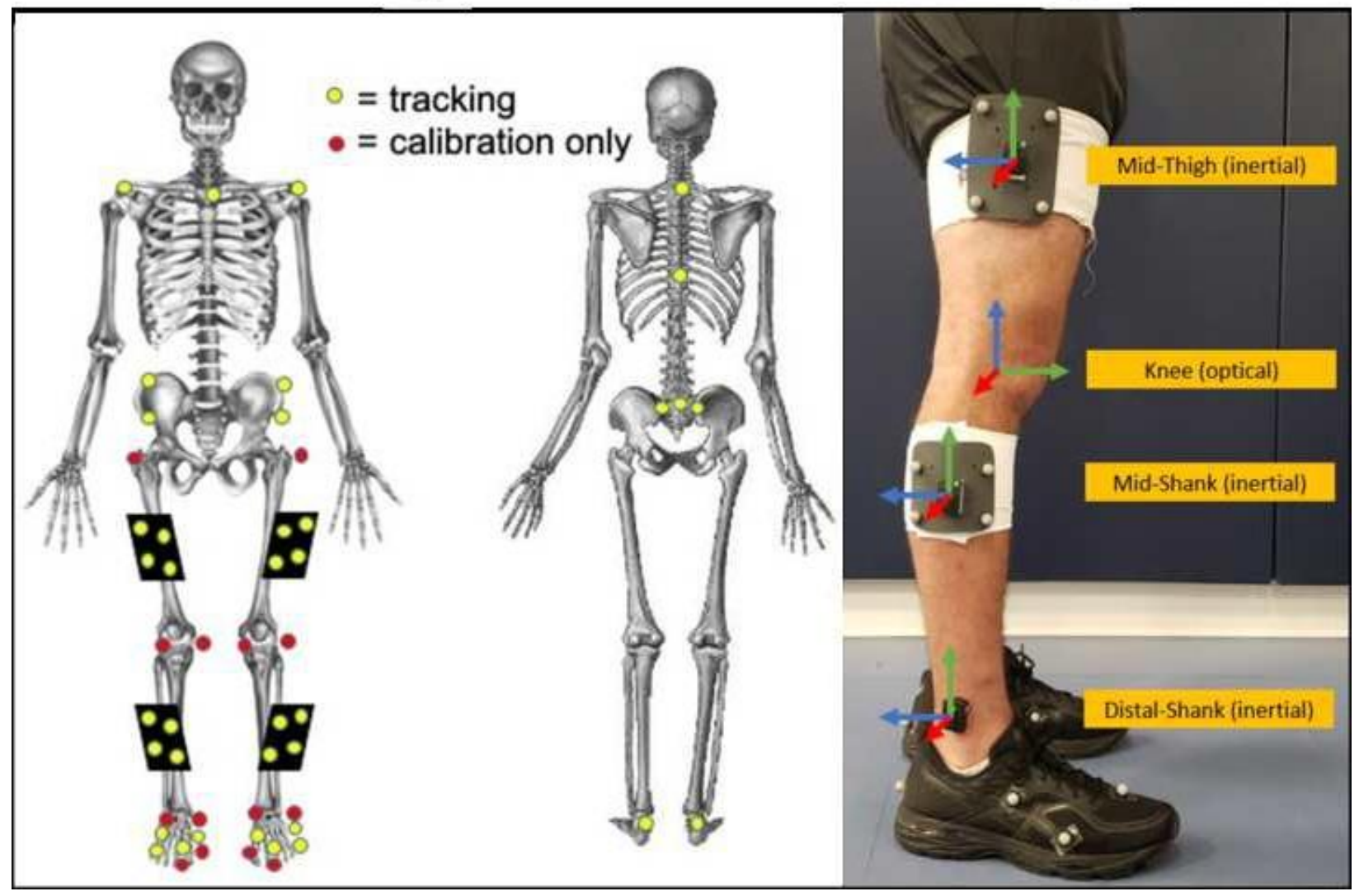

455 Figure 1. (A) Optical motion capture markers (red markers used for static trial only) and (B) inertial

456 sensors, showing placement of the mid-thigh and mid-shank inertial sensors on the optical motion

457 capture marker clusters for the thigh and shank segments, respectively, and distal shank inertial sensor

458 directly on the skin on the lateral aspect of the distal tibia, along with the coordinate systems for the

459 knee (optical system) and inertial sensors. 

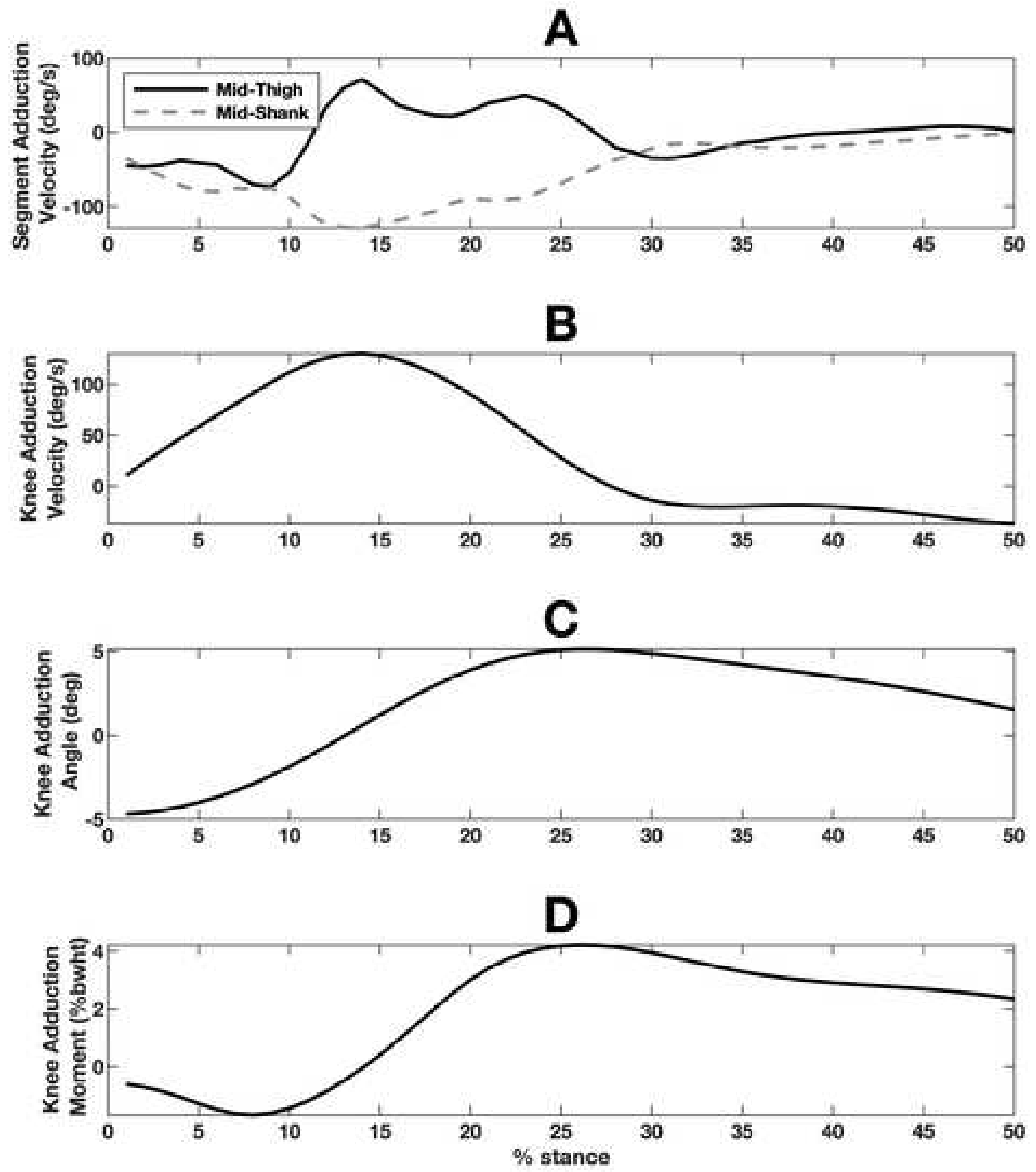

461 Figure 2. Representative waveforms from self-selected speed walking showing (A) inertial sensor

462 segment adduction velocity from the mid-thigh and mid-shank sensors, (B) knee adduction velocity, (C)

463 knee adduction angle and knee adduction excursion, and (D) external knee adduction moment (EKAM). 


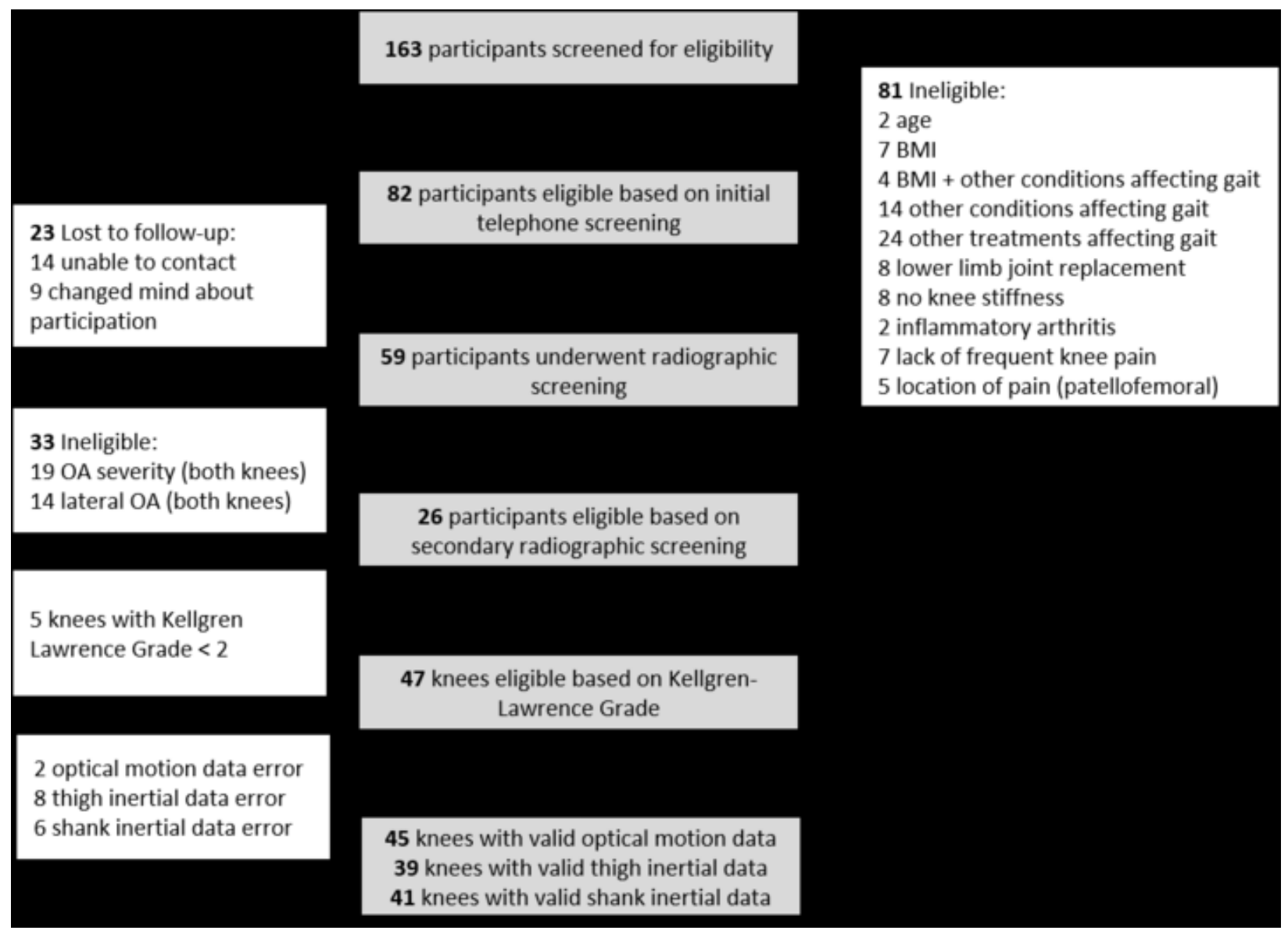

465 Figure 3. Study recruitment and final study sample. 

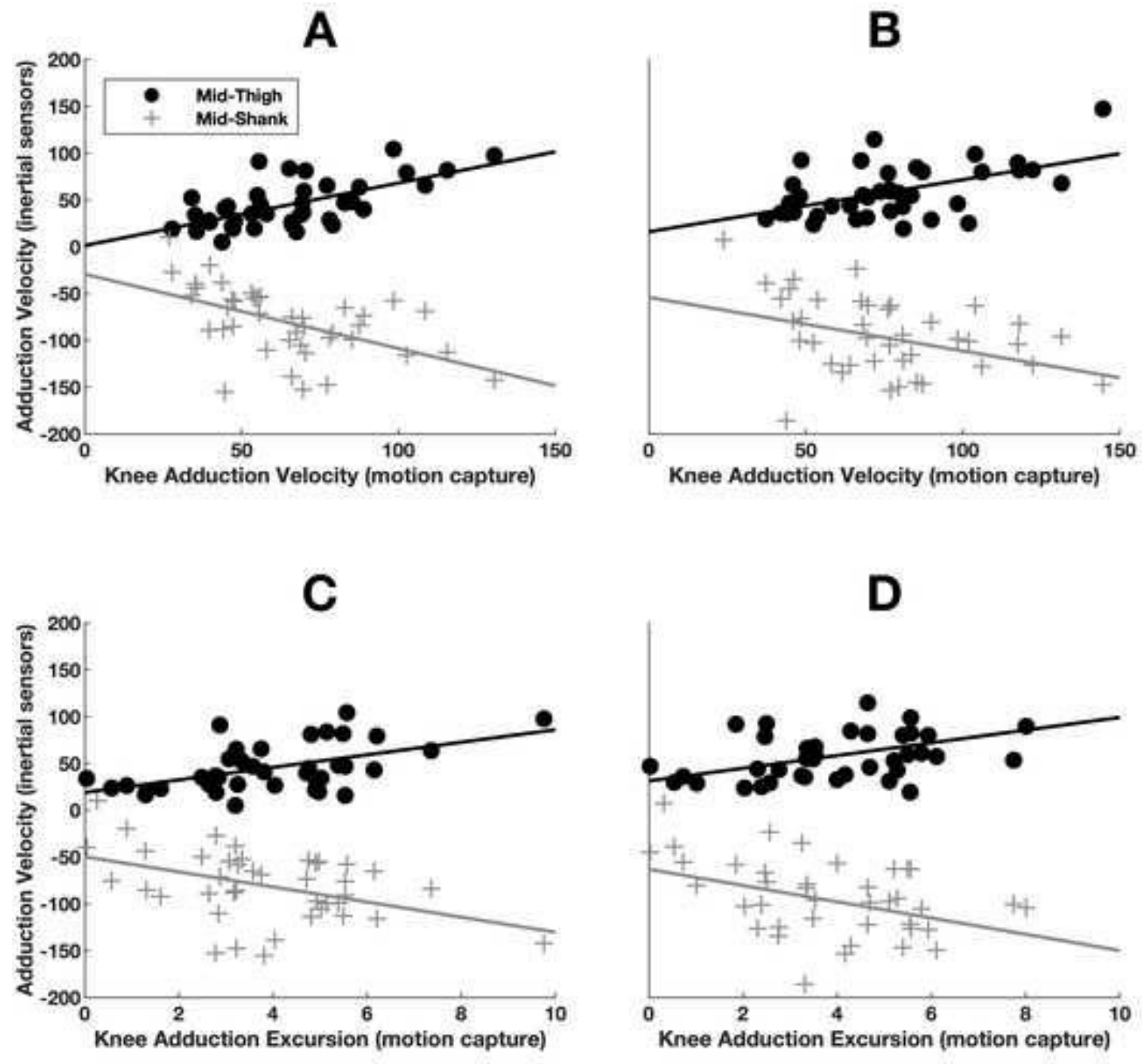

Figure 4. Relationships between segment adduction velocity (captured by the inertial sensors) and (A) knee adduction velocity during self-selected speed walking, (B) knee adduction velocity during fast speed walking, (C) knee adduction excursion during self-selected speed walking, and (D) knee adduction excursion during fast speed walking in knees with radiographic osteoarthritis. 